\title{
Modular Invariance for Interacting Bosonic Strings at Finite Temperature
}

\author{
Bruce McClain ${ }^{1}$ and Bernard David Barkan Roth ${ }^{2}$ \\ 1 Department of Physics, University of Utah, Salt Lake City, Utah 84112, USA \\ 2 Theory Group, Department of Physics, University of Texas Austin, Texas 78712, USA
}

\begin{abstract}
We study the finite temperature string path integral introduced by Polchinski [1]. It is shown that on an arbitrary genus world sheet all windings of the fields around the compact time direction can be rotated into a single cycle. The modular invariance of this result is demonstrated.
\end{abstract}

\section{Introduction}

The recent work [2] on strings at finite temperature has so far considered free strings in flat spacetime. Since the extended structure of strings will only become apparent near the Planck scale, the most likely setting for obtaining (indirect) evidence is the observation of cosmological effects from the early universe. A most remarkable property is the existence of the Hagedorn limiting temperature [3] at which the free energy diverges. Although this critical temperature has been shown only in the ideal gas approximation, it signals the likelihood that string cosmology differs drastically from the standard big bang scenario in the early stages.

During the Planck epoch it is impossible to ignore the extreme conditions which exist; it is expected that any perturbative expansion will then be invalid. Nevertheless, it is still interesting to consider the interactions of strings in a regime where perturbation theory holds. It is therefore the aim of this paper to show how to include interactions for the closed bosonic string in 26 dimensions. For simplicity, curvature effects will not be considered. In this regard, our result should be considered more as a lowest order contribution for more general backgrounds when an adiabatic approximation is used. The starting point will be the Polyakov path integral as discussed by Polchinski [1].

In [1], the free energy density $F(\beta)$ is calculated for a gas of a variable number of non-interacting closed strings. In other approaches [2], which focus on calculating the Hagedorn temperature, the partition function for a single string (in a heat bath) is derived as for a system of independent oscillators. With the inclusion of interactions, though, a more systematic treatment is needed.

The requirement of modular invariance of physical quantities - here, the free energy - shall play a key role in this formulation. In particular, the embeddings of a 
compact Riemann surface $M_{\mathrm{g}}$, of genus $\mathrm{g}$, into a Euclidean spacetime periodic in time $\mathbb{R}^{25} \times S^{1}$, can be characterized by a single winding number. This result is nonobvious in that each handle on $M_{\mathrm{g}}$ can be given two independent winding numbers corresponding to each of its homotopically (and homologically) inequivalent cycles. It will be shown that a sequence of $S p(2 \mathrm{~g}, \mathbb{Z})$ symplectic modular transformations acting on the homology basis can be chosen so that the embedded surface $X^{\mu}(z, \bar{z})$ has all non-trivial windings in only one homology cycle ${ }^{1}$.

This may provide two technical advantages. For one, summation over only one variable should be easier than over $2 g$ variables. In addition, the integration region in Teichmüller space should be simplified somewhat (see Sect. III). For genus $\mathbf{g}>3$, the fundamental region $F$ in Teichmüller space does not admit a tractable parameterization. As in the case discussed by Polchinski, elaborated upon in Sect. II, the integration region can be chosen larger than $F$ at the expense of dropping manifest modular invariance. It will be shown that $F(\beta)$ is, nonetheless, modular invariant, whatever the form.

As a starting point, the genus one case will be examined in detail. Imposing "symmetric" boundary conditions on the embedding of the torus preserves explicit modular invariance for the free energy. This form of the free energy (15) will be shown to be equivalent to that derived in [1] with "asymmetric" boundary conditions in which modular invariance is not manifest (9). As a consistency check on the path integral method, the critical temperature is obtained in Appendix AI. Divergences are examined in a second Appendix AII.

The generalization to higher genus surfaces requires somewhat more mathematical structure. The solutions $X^{\mu}(z, \bar{z})$ which satisfy the appropriate symmetric boundary conditions depend on the abelian differentials on $M_{\mathrm{g}}$. The winding number contribution to $F(\beta)$ appears only in the exponentiated classical action through terms depending on the period matrix $\Omega$. The proof of modular invariance depends, then, on the transformation properties of these objects.

The free energy can be written as an integral over the modular parameters for $M_{\mathrm{g}}$, with sums over winding numbers. Modular transformations correspond to changing the dummy summation variables (the individual winding numbers) and leaving the remainder of the integrand invariant.

The path integral for the connected vacuum-to-vacuum amplitude, for interacting bosonic strings in flat Euclidean spacetime, can be written as a sum over contributions of surfaces $M_{\mathrm{g}}$ for every genus:

$$
\begin{aligned}
W=-\ln Z=\sum_{\mathrm{g}=1}^{\infty} W_{\mathrm{g}} \equiv \sum_{\mathrm{g}} \int_{\frac{\left[d \mathrm{~g}_{a b}\right]\left[d X^{\mu}\right]}{V_{G C} V_{W}}} \\
\cdot \exp -\int_{M_{\mathrm{g}}} d^{2} z \sqrt{\mathrm{g}}\left[\frac{T}{2} \mathrm{~g}^{a b} \partial_{a} X^{\mu} \partial_{b} X^{\mu}+\lambda R+\mu^{2}\right]
\end{aligned}
$$

The volumes of the general coordinate and Weyl groups have been divided out, leaving an integral over two dimensional metrics parameterized by the Teichmüller

1 G. Moore has stated this result for genus 2[4], and has an unpublished proof for this case 
(modular) parameters of $M_{\mathrm{g}}$. In Euclidean spacetimes, the integration over $X^{\mu}$ is gaussian. The contribution of the scalar curvature of $M_{\mathrm{g}}$ weights each term $W_{\mathrm{g}}$ by appropriate powers of the string coupling constant (suppressed throughout) and $\mu^{2}$ is chosen in $d=26$ to cancel the conformal anomaly. $T$ is the string tension. Temperature will be denoted by its inverse, $\beta$. For reviews of the mathematical concepts, see $[5,6]$.

The free energy is defined by

$$
e^{-\beta F(\beta)}=\operatorname{Tr} e^{-\beta \hat{H}}
$$

In the path integral language, this becomes

$$
F(\beta)=\sum_{\mathrm{g}=1}^{\infty} F_{\mathrm{g}}(\beta) \equiv \sum_{\mathrm{g}} \int_{\mathfrak{M}_{\mathrm{g}} \times \mathscr{E} / \mathrm{D}_{\text {Iff }} M_{\mathrm{g}} \ltimes W}\left[d \mathrm{~g}_{a b}\right]\left[d X^{\mu}\right] e^{-S[\mathrm{~g} \cdot X]} .
$$

The integral $\left[d g_{a b}\right]$ is over $\mathfrak{M}_{\mathrm{g}}$, the space of all metrics on the string world sheet $M_{\mathrm{g}}$, modulo gauge transformations in the semi-direct product of the diffeomorphism and Weyl groups. Because the trace in (2) identifies initial states with final states [7], the path integral (3) involves only compact world sheets. So topology is determined completely by the genus. (Actually, the scalar curvature term in (1) integrates to the Euler character which determines the topology uniquely for the boundaryless surfaces of interest here.) The sum over $\mathrm{g}$ is a loop expansion; interactions are included by adding handles to the world sheet.

The integral $\left[d X^{\mu}\right]$ is taken over the space $\mathscr{E}$ of all embeddings of $M_{\mathrm{g}}$ into spacetime. The trace (2), together with the fact that the hamiltonian $\hat{H}$ is a timetranslation operator, forces the identification of the field $X^{0}(z, \bar{z})$ with $X^{0}(z, \bar{z})+\beta$. Therefore the embeddings are into a spacetime with periodic time coordinate, $X^{\mu}$ : $M_{\mathrm{g}} \rightarrow \mathbb{R}^{25} \times S^{1}$. The space of embeddings decomposes as $\mathscr{E}=\bigoplus_{\vec{m}, \vec{n}} \mathscr{E}(\vec{m}, \vec{n})$, where $m_{i}$, $n_{i} \in \mathbb{Z}(i=1, \ldots, \mathrm{g})$ are the winding numbers of $X^{0}(z, \bar{z})$ around $S^{1}$ as its argument moves around the homology cycles $a_{i}, b_{i}$ (see Sect. III).

Section II details how this works for the case $g=1$. Section III develops the mathematical machinery needed to handle arbitrary $g$ and contains the proof of our main result. Conclusions and speculations are contained in the final section.

\section{Modular Invariance for Genus One}

To provide a setting for the treatment of arbitrary genus Riemann surfaces while maintaining explicit modular invariance, the genus one result [1] will be reviewed. Invariance under $S p(2, \mathbb{Z})=S L(2, \mathbb{Z})$ will be shown by construction. The notation of [1] will be followed with slight modifications for later convenience.

To calculate $W_{1}$, from which the one loop vacuum energy density is obtained, one must specify boundary conditions on $\mathrm{g}_{a b}(\sigma)$ and $X^{\mu}(\sigma)$. As usual for the metric on the torus,

$$
\mathrm{g}_{a b}\left(\sigma_{1}+1, \sigma_{2}\right)=\mathrm{g}_{a b}\left(\sigma_{1}, \sigma_{2}\right)=\mathrm{g}_{a b}\left(\sigma_{1}, \sigma_{2}+1\right)
$$

After a general coordinate transformation, all variations in the metric, up to Weyl 
rescalings, are embodied in the complex Teichmüller parameter, $\tau=\tau_{1}+i \tau_{2}$ :

$$
d s^{2}=e^{\phi(\sigma)}\left|d \sigma_{1}+\tau d \sigma_{2}\right|^{2}, \quad \tau_{2}>0 .
$$

The embedding coordinates $X^{\mu}(\sigma)$ are simply periodic functions, as in (4).

To obtain $F_{1}(\beta)$ instead of $W_{1}$, the boundary conditions on $X^{0}(z, \bar{z})$ must be modified. Polchinski chose the boundary conditions

$$
\begin{aligned}
& X^{\mu}\left(\sigma_{1}, \sigma_{2}+1\right)=X^{\mu}\left(\sigma_{1}, \sigma_{2}\right)+r \beta \delta_{0}^{\mu}, \quad r \in \mathbb{Z}, \\
& X^{\mu}\left(\sigma_{1}+1, \sigma_{2}\right)=X^{\mu}\left(\sigma_{1}, \sigma_{2}\right) .
\end{aligned}
$$

The fields $X^{\mu}(\sigma)$ can then be decomposed into a periodic piece and a linear piece,

$$
X^{\mu}\left(\sigma_{1}, \sigma_{2}\right)=Y^{\mu}\left(\sigma_{1}, \sigma_{2}\right)+r \beta \sigma_{2} \delta_{0}^{\mu}
$$

for which the path integration measure remains unchanged, $\left[d Y^{\mu}\right]=\left[d X^{\mu}\right]$. Therefore, the only modification to $W_{1}$ from the zero temperature result is in the additional term in the classical action functional coming from the winding number term in (7). Thus

$$
S[X, \mathrm{~g}]=S[Y, \mathrm{~g}]+\frac{r^{2} \beta^{2} T}{2 \tau_{2}},
$$

where $S[Y, \mathrm{~g}]$ is the usual Polyakov action as contained in (1). The integrand in $W_{1}$ is otherwise unchanged. After dividing out the volume of spacetime, the free energy density is obtained:

$$
\begin{aligned}
& F_{1}(\beta)=-T^{13} \int_{S} d^{2} \tau \frac{1}{4 \pi \tau_{2}^{2}} I_{1}(\tau) \sum_{r=-\infty}^{\infty} \exp -\frac{r^{2} \beta^{2} T}{2 \tau_{2}}, \\
& I_{1}(\tau) \equiv e^{4 \pi \tau_{2}}\left(2 \pi \tau_{2}\right)^{-12}\left|f\left(e^{2 \pi i \tau}\right)\right|^{-48}
\end{aligned}
$$

In (9), the integrand $I_{1}(\tau)$ is identical to that in the calculation of the cosmological constant $\Lambda$ [1]. The prime on the sum excludes the $r=0$ term (related to $\Lambda$ ). The integration in Teichmüller space is over the strip, $S$, defined by $\tau_{2}>0,-\frac{1}{2}<\tau_{1}<\frac{1}{2}$. The function $f$ is discussed in Appendix I.

In general, the torus is invariant under the group of modular transformations generated by (i) translations $\sigma_{1} \rightarrow \sigma_{1}+\sigma_{2}$ and $\sigma_{2} \rightarrow \sigma_{2}$, corresponding to $\tau \rightarrow \tau+1$, and (ii) inversions $\sigma_{1} \rightarrow \sigma_{2}$ and $\sigma_{2} \rightarrow-\sigma_{1}$, corresponding to $\tau \rightarrow-1 / \tau$. However, the boundary conditions (6) respect only translation invariance. Therefore, the integration in (9) is over the quotient of Teichmüller space $\mathbb{C}_{+}$, the upper half complex plane, by the translation subgroup of $\operatorname{Sp}(2, \mathbb{Z})$, denoted $\Gamma_{t}$. That is, $S=\mathbb{C}_{+} / \Gamma_{t}$.

Full modular invariance is made explicit by altering the boundary conditions (6) to

$$
\begin{aligned}
& X^{\mu}\left(\sigma_{1}+1, \sigma_{2}\right)=X^{\mu}\left(\sigma_{1}, \sigma_{2}\right)+r_{1} \beta \delta_{0}^{\mu}, \\
& X^{\mu}\left(\sigma_{1}, \sigma_{2}+1\right)=X^{\mu}\left(\sigma_{1}, \sigma_{2}\right)+r_{2} \beta \delta_{0}^{\mu}, \quad r_{1}, r_{2} \in \mathbb{Z} .
\end{aligned}
$$

These are solved by

$$
X^{\mu}\left(\sigma_{1}, \sigma_{2}\right)=Y^{\mu}\left(\sigma_{1}, \sigma_{2}\right)+r_{1} \beta \sigma_{1} \delta_{0}^{\mu}+r_{2} \beta \sigma_{2} \delta_{0}^{\mu},
$$

where now the modification to the classical action is not a simple generalization of 
(8):

$$
S[X, \mathrm{~g}]=S[Y, \mathrm{~g}]+\frac{\beta^{2} T}{2 \tau_{2}}\left(|\tau|^{2} r_{1}^{2}+r_{2}^{2}-2 r_{1} r_{2} \tau\right) .
$$

For future convenience, it is helpful to define

$$
E\left(\tau, r_{1}, r_{2}\right) \equiv|\tau|^{2} r_{1}^{2}+r_{2}^{2}-2 r_{1} r_{2} \tau
$$

The free energy can then be written

$$
F_{1}(\beta)=-T^{13} \int_{F} d^{2} \tau \frac{1}{4 \pi \tau_{2}^{2}} I_{1}(\tau) \sum_{r_{1}, r_{2}=-\infty}^{\infty} \exp -\frac{\beta^{2} T}{2 \tau_{2}} E\left(\tau, r_{1}, r_{2}\right) .
$$

The $r_{1}=r_{2}=0$ term is excluded by the prime.

It is easy to check that invariance under $\operatorname{Sp}(2, \mathbb{Z})$ is manifest. Under translations (i) $\tau \rightarrow \tau+1$, the summation variables change by $r_{1} \rightarrow r_{1}, r_{2} \rightarrow r_{2}-r_{1}$. Under inversions (ii) $\tau \rightarrow-1 / \tau, r_{1} \rightarrow r_{2}, r_{2} \rightarrow-r_{1}$. The prime on the sum is understood to be applied after the change of summation variables, corresponding to the subtraction of the infinite zero point energy. Therefore, the modular group acts on the winding number terms as a change in dummy variables. The remainder of the integrand, $I_{1}(\tau)$, together with the measure $d^{2} \tau\left(1 / 4 \pi \tau_{2}^{2}\right)$ is separately modular invariant, as in the zero temperature case. Since (15) is explicitly modular invariant, the integration region $F$ is the fundamental domain in Teichmüller space, $F=\mathbb{C}_{+} / \operatorname{Sp}(2, \mathbb{Z}), \tau_{2}>0$, $-\frac{1}{2}<\tau_{1}<\frac{1}{2}|\tau|>1$, called moduli space. This is the well-known "keyhole" shape.

It can now be shown that (9) and (15) are actually equal. First, observe that a fractional linear transformation can be found, $\gamma: \tau \rightarrow \tau^{\prime}$, such that $1 / \tau_{2} E\left(\tau, r_{1}, r_{2}\right) \rightarrow$ $1 / \tau_{2}^{\prime} r^{2} \equiv\left[\operatorname{Im}\left(\gamma\left(r_{1}, r_{2}\right) \circ \tau\right)\right]^{-1} r^{2}$, where $\gamma\left(r_{1}, r_{2}\right)$ is a $2 \times 2$ matrix representative of a particular $S p(2, \mathbb{Z})$ group element. Suppose, initially, that $r_{1}$ and $r_{2}$ are relatively prime. Then there exist relatively prime integers, $a$ and $b$, such that $\gamma\left(r_{1}, r_{2}\right)=$ $\left(\begin{array}{ll}a & b \\ r_{1} & r_{2}\end{array}\right), \operatorname{det}\left(\begin{array}{ll}a & b \\ r_{1} & r_{2}\end{array}\right)=1$, and such that $\tau_{2}^{\prime}=\operatorname{Im} \tau^{\prime}=\operatorname{Im}\left(a \tau+b / r_{1} \dot{\tau}+r_{2}\right)=$ $\tau_{2} E\left(\tau, r_{1}, r_{2}\right)^{-1}$. That is, the integers $a, b$ do not occur explicitly in $\operatorname{Im} \tau^{\prime}$, although they do occur in $\operatorname{Re} \tau^{\prime}$. If $r_{1}, r_{2}$ are not relatively prime, let $r$ be their greatest common divisor, so $r_{1}=r \rho_{1}, r_{2}=r \rho_{2}$, where $\rho_{1}, \rho_{2}$ are relatively prime. Then $E\left(\tau, r_{1}, r_{2}\right)=r^{2} E\left(\tau, \rho_{1}, \rho_{2}\right)$ and a matrix $\gamma\left(\rho_{1}, \rho_{2}\right)=\left(\begin{array}{ll}a & b \\ \rho_{1} & \rho_{2}\end{array}\right)$ can be found such that $\tau_{2}^{\prime}=\tau_{2} E\left(\tau, \rho_{1}, \rho_{2}\right)^{-1}$.

The integers $r, \rho_{1}, \rho_{2}$ have a physical interpretation. For each $r_{1}, r_{2}$ in the double sum, $\rho_{1}$ and $\rho_{2}$ determine a linear combination of $\sigma_{1}$ and $\sigma_{2}$ which selects out a time direction, $\sigma_{0}$, around the torus. The integer $r$ is a topological invariant which counts the number of times $X^{0}$ is circumnavigated in going once around the path $\sigma_{0}$. The important point is that $r_{1}$ and $r_{2}$ individually do not contain topological information, so it is possible to put all the winding in either the $\sigma_{1}$ or $\sigma_{2}$ cycle. This will generalize at higher genus.

Continuing the proof, Teichmüller space consists of an infinite number of domains, $F_{\gamma}$, which are related to $F$ by the action of $\operatorname{Sp}(2, \mathbb{Z})$ elements $\gamma\left(\rho_{1}, \rho_{2}\right)$. 
Furthermore, each domain $F_{\gamma}$ is obtained from $F$ by a unique $\gamma \in S p(2, \mathbb{Z})$. Thus

$$
\begin{aligned}
& \int_{F} d^{2} \tau \frac{1}{4 \pi \tau_{2}^{2}} I_{1}(\tau) \sum_{r_{1}, r_{2}} \exp -\frac{\beta^{2} T}{2 \tau_{2}} E\left(\tau, r_{1}, r_{2}\right) \\
& =\int_{F} d^{2} \tau \frac{1}{4 \pi \tau_{2}^{2}} I_{1}(\tau) \sum_{\rho_{1}, \rho_{2}} \sum_{r} \exp -\frac{\beta^{2} T}{2 \tau_{2}} r^{2} E\left(\tau, \rho_{1}, \rho_{2}\right) \\
& =\sum_{r} \sum_{\rho_{1}, \rho_{2}} \int_{F} d^{2} \tau I_{1}(\tau) \exp -\frac{\beta^{2} T}{2}\left[\operatorname{Im}\left(\gamma\left(\rho_{1}, \rho_{2}\right) \circ \tau\right)\right]^{-1} r^{2},
\end{aligned}
$$

where $\gamma$ is chosen from the equivalence class of modular transformations, related by translations, such that $\gamma: \tau \rightarrow \tau^{\prime}$ as needed to recover (9). Indeed, if $\gamma=\left(\begin{array}{ll}a & b \\ \rho_{1} & \rho_{2}\end{array}\right)$ is such an element of $\operatorname{Sp}(2, \mathbb{Z})$, then so is a translation, $\gamma^{\prime}=\left(\begin{array}{ll}1 & n \\ 0 & 1\end{array}\right) \cdot\left(\begin{array}{ll}a & b \\ \rho_{1} & \rho_{2}\end{array}\right)=$ $\left(\begin{array}{cc}a+n \rho_{1} & b+n \rho_{2} \\ \rho_{1} & \rho_{2}\end{array}\right)$. It is therefore possible to choose each $\gamma$ so that $\gamma \circ \tau$ lies in a domain $F_{\gamma}$ occupying the same strip as the fundamental domain $F$.

Defining $\Gamma \equiv S p(2, \mathbb{Z})$, the sum over relative primes can be converted to a sum over $\gamma\left(\rho_{1}, \rho_{2}\right)$ in the coset space $\Gamma / \Gamma_{t}$ :

$$
\sum_{r} \sum_{\rho_{1}, \rho_{2}} \int_{\tau \in F} \rightarrow \sum_{r} \sum_{\gamma \in \Gamma / \Gamma_{t}} \int_{\gamma_{0} \tau \in F_{\gamma}}
$$

The sum over $\gamma$ then acts to sum over all "copies" of $F$ in the strip, $S$.

$$
\sum_{\gamma \in \Gamma / \Gamma_{t}} \int_{F_{\gamma}} \rightarrow \int_{\tau^{\prime} \in S=\mathbb{C}_{+} / \Gamma_{t}}
$$

To complete the proof, note that $I_{1}(\tau)$ with the measure $d^{2} \tau\left(1 / 4 \pi \tau_{2}^{2}\right)$ is separately modular invariant, coming from the zero temperature result [1]. It follows that (9) and (15) are equivalent by virtue of

$$
\begin{gathered}
\int_{F} d^{2} \tau \frac{1}{4 \pi \tau_{2}^{2}} I_{1}(\tau) \sum_{r_{1}, r_{2}}^{\prime} \exp -\frac{\beta^{2} T}{2 \tau_{2}} E\left(\tau, r_{1}, r_{2}\right) \\
=\int_{S} d^{2} \tau^{\prime} \frac{1}{4 \pi \tau_{2}^{\prime 2}} I_{1}\left(\tau^{\prime}\right) \sum_{r}^{\prime} \exp -\frac{\beta^{2} T}{2 \tau_{2}^{\prime}} r^{2} .
\end{gathered}
$$

Guided by this explicit construction, the higher genus case can be tackled, but only after the introduction of more mathematical machinery.

\section{Free Energy for Interacting Strings}

The path integral (3) sums over all winding numbers for the embedding of handles of $M_{\mathrm{g}}$ around the periodic coordinate of spacetime, $X^{0}$. This can be done in several ways. To maintain explicit modular invariance requires summing over winding numbers for each non-trivial homology cycle. Alternatively, one may sacrifice the explicit invariance by (arbitrarily) choosing one non-trivial loop on $M_{\mathrm{g}}$ as the "time" direction and summing over windings of this loop only. 
In the first case, $F_{\mathrm{g}}(\beta)$ is expressed as an integral over modular space-the quotient of Teichmüller space $\mathscr{T}$ by the modular group $\Gamma_{\mathrm{g}} \subset S p(2 \mathrm{~g}, \mathbb{Z})$. In the second instance, the integration region corresponds to $\mathscr{T}$ modulo that subgroup of $\Gamma_{\mathrm{g}}$ which preserves the arbitrarily chosen "time" loop on the worldsheet. This may have the advantage of yielding more tractable modular integrals. It does not, however, manifest the modular invariance of $F_{\mathrm{g}}(\beta)$. The equivalence of these formulations will be demonstrated below.

Following [8-10], choose a canonical homology basis $a_{i}, b_{i}(i=1, \ldots, \mathrm{g})$ on $M_{\mathrm{g}}$. The winding numbers $m_{i}, n_{i}$ count the number of times $X^{0}(z, \bar{z})$ wraps around $S^{1}$ as $z$ traverses $a_{i}, b_{i}$. The space of abelian differentials of the first type is defined as the set of closed holomorphic 1-forms modulo exact holomorphic 1-forms. The RiemannRoch theorem states that this space is g-dimensional on a genus $g$ surface. A normalized basis $\omega_{i}$ for this space can be defined by

$$
\int_{a_{i}} \omega_{j}=\delta_{i j} \text {. }
$$

This completely determines the basis $\omega_{i}$. The period matrix $\Omega$ is defined by

$$
\int_{b_{i}} \omega_{j}=\Omega_{i j}
$$

The period matrix is symmetric, $\Omega_{i j}=\Omega_{j i}$, and has positive imaginary part, $\operatorname{Im} \Omega>0$. In addition, $\Omega$ is a holomorphic function of the moduli; in fact, this can be used as the definition of the complex structure on moduli space [9-11].

It is convenient to introduce the jacobi maps $\phi_{i}(z)$

$$
\phi_{i}(z)=\int_{z_{0}}^{z} \omega_{i}
$$

where $z_{0}$ is a fixed, arbitrary point in $M_{\mathrm{g}}$. These maps are not well-defined maps of $M_{\mathrm{g}} \rightarrow \mathbb{C}^{\mathrm{g}}$; the integral (22) depends on the homology class of the path from $z_{0}$ to $z$. For example,

$$
\phi_{i}^{\prime}(z)=\int_{z_{0}}^{z} \omega_{i}+\oint_{a_{J} \text { or } b_{j}} \omega_{i} \neq \phi_{i}(z)
$$

As $z$ traverses $a_{j}, \phi_{i} \rightarrow \phi_{i}+\delta_{i j}$, while if $z$ traverses $b_{j}$, then $\phi_{i} \rightarrow \phi_{i}+\Omega_{i j}$. The Jacobi map is well-defined, however, as a map $\vec{\phi}: M_{\mathrm{g}} \rightarrow \mathbb{C}^{\mathrm{g}} / L_{\Omega}$, where $L_{\Omega}$ is the lattice spanned by $\Omega_{i j}$; i.e., $\vec{z} \simeq \vec{z}^{\prime}$ if $\vec{z}^{\prime}=\vec{z}+\vec{m}+\Omega \cdot \vec{n}$.

The boundary conditions on $X^{0}(z, \bar{z})$ in (3) can be implemented using the Jacobi maps. Following (11), introduce the ansatz

$$
\begin{gathered}
X^{\mu}(z, \bar{z})=Y^{\mu}(z, \bar{z})+\Delta X(z, \bar{z}) \delta_{0}^{\mu}, \\
\Delta X(z, \bar{z})=\sum_{i=1}^{\mathrm{g}}\left[H_{i} \phi_{i}(z)+\bar{H}_{i} \bar{\phi}_{i}(\bar{z})\right] .
\end{gathered}
$$

$Y^{\mu}(z, \bar{z})$ has zero winding number around all homology cycles. The coefficients $H_{i}$ depend on $\beta$, the winding numbers and $\Omega . \Delta X$ trivially satisfies Laplace's equation,

$$
\partial_{z} \partial_{\bar{z}} \Delta X(z, \bar{z})=0
$$

since $\Delta X$ is the sum of a holomorphic function and its complex conjugate. As $z$ 
traverses $a_{i}, X^{0}$ changes by $\beta m_{i}$. From (22) and (24) one finds that

$$
\operatorname{Re} H_{i}=\beta m_{i} \text {. }
$$

Similarly, letting $z$ traverse $b_{i}$ determines

$$
\operatorname{Re} H_{j} \Omega_{j i}=\beta n_{i} \text {. }
$$

Equations (26) and (27) determine $H_{j}$ uniquely:

$$
H_{j}=\frac{i}{2} \beta(\operatorname{Im} \Omega)_{j k}^{-1}\left[\bar{\Omega}_{k l} m_{l}-n_{k}\right]
$$

To proceed further requires the introduction of a metric. It is conventional to work with a constant negative curvature metric with $R_{(2)}=-1$. After canonical dissection, $M_{\mathrm{g}}$ can be represented as $H / \mathscr{F}_{\mathrm{g}}$, where $H$, the hyperbolic plane, is the universal covering space of $M_{\mathrm{g}}$ and $\mathscr{F}_{\mathrm{g}}$ is the Fuchsian group of $M_{\mathrm{g}}$. The natural complex coordinate $z$ in $H$ can then be used to label points in $M_{\mathrm{g}}$. In these coordinates the metric is

$$
d s^{2}=(\operatorname{Im} z)^{-2} d z d \bar{z} .
$$

Substituting (23), (24), (28) and (29) into the action (1) results in

$$
\begin{gathered}
S[X, \mathrm{~g}]=X[Y, \mathrm{~g}]+\Delta S(\beta ; \vec{m}, \vec{n}), \\
\Delta S(\beta ; \vec{m}, \vec{n})=\frac{\beta^{2} T}{2}\left[m_{l} \Omega_{l i}-n_{i}\right](\operatorname{Im} \Omega)_{i j}^{-1}\left[\bar{\Omega}_{j k} m_{k}-n_{j}\right],
\end{gathered}
$$

where (20), (21) and (25) have been used.

Since $[d Y]=[d X]$, the Gaussian integral over $Y^{\mu}$ can be performed in (3) yielding (Det $\left.\Delta_{g}\right)^{-13}$, where $\Delta_{g}$ is the scalar Laplacian on $M_{\mathrm{g}}$. Gauge degrees of freedom are eliminated by the Fadeev-Popov determinant Det $P^{+} P$. The integral $\left[d g_{a b}\right]$ then reduces to an integral over the moduli space $\mathscr{M}$, the quotient of Teichmüller space $\mathscr{T}$ by the modular group $\Gamma_{g}$, thereby giving

$$
\begin{aligned}
F_{\mathrm{g}}(\beta) & =\sum_{m_{\imath}, n_{l}=-\infty}^{\infty} \int_{\mathscr{M}}(d \tau)_{\mathrm{WP}}\left(\operatorname{Det} P^{+} P\right)^{1 / 2}\left(\operatorname{Det} \Delta_{\mathrm{g}}\right)^{-13} e^{-\Delta S(\beta ; \vec{m}, \vec{n})} \\
& \equiv \sum_{\vec{m}, \vec{n}}^{\prime} \int_{\mathscr{M}}(d \tau) I_{\mathrm{g}}(\tau) e^{-\Delta S(\beta, \vec{m}, \vec{n})}
\end{aligned}
$$

Here $(d \tau)_{\text {WP }}$ denotes the Weil-Petersson measure on $\mathscr{M}$. The prime on the sum indicates that the $m_{i}=n_{i}=0$ term, corresponding to the zero temperature vacuum energy density, has been subtracted. The factors $(d \tau)_{\mathrm{WP}},\left(\operatorname{Det} \Delta_{g}\right)$ and $\operatorname{Det}\left(P^{+} P\right)$ are each individually modular invariant. The invariance of $\sum_{\vec{m}, \vec{n}} e^{-\Delta S}$ will be checked explicitly. For future convenience, we have defined $I_{g}(\tau) \equiv\left(\stackrel{\vec{m}, \vec{n}}{\operatorname{Det}} \Delta_{g}\right)^{-13}\left(\operatorname{Det} P^{+} P\right)^{1 / 2}$.

Modular transformations are defined to be those diffeomorphisms, not deformable to the identity, which take $M_{\mathrm{g}}$ to itself. An example of such a transformation is the Dehn twist: Cut $M_{\mathrm{g}}$ along any homotopically non-trivial closed curve, twist one side of the cut by $2 \pi$ relative to the other side, and then rejoin the edges. It is clear that any Dehn twist is a disconnected diffeomorphism. The converse is also true. 
Any modular transformation can be represented as a sequence of Dehn twists. Moreover, a finite number of Dehn twists generates the entire modular group.

Under a Dehn twist, a canonical homology basis $(\vec{a}, \vec{b}) \equiv\left(a_{1}, \ldots, a_{\mathrm{g}}, b_{1}, \ldots, b_{\mathrm{g}}\right)$ changes to $\left(\vec{a}^{\prime}, \vec{b}^{\prime}\right)$, where $\vec{a}^{\prime}$ and $\vec{b}^{\prime}$ are linear combinations of $\vec{a}$ and $\vec{b}$ with integer coefficients.

$$
\left(\begin{array}{c}
\vec{a} \\
\vec{b}
\end{array}\right) \rightarrow\left(\begin{array}{c}
\vec{a}^{\prime} \\
\vec{b}^{\prime}
\end{array}\right)=\left(\begin{array}{ll}
A & B \\
C & D
\end{array}\right)\left(\begin{array}{c}
\vec{a} \\
\vec{b}
\end{array}\right)
$$

The matrix $\left(\begin{array}{ll}A & B \\ C & D\end{array}\right)$ has block entries which are $\mathrm{g} \times \mathrm{g}$ matrices with integer elements. This allows a matrix representation of the Dehn twists, which are known to preserve the (signed) intersection numbers of any two curves on $M_{\mathrm{g}}$. For the basis $(\vec{a}, \vec{b})$, the intersection matrix is $\left(\begin{array}{rr}0 & 1 \\ -1 & 0\end{array}\right)$ in block form. Thus

$$
\left(\begin{array}{ll}
A & B \\
C & D
\end{array}\right)^{T}\left(\begin{array}{rr}
0 & 1 \\
-1 & 0
\end{array}\right)\left(\begin{array}{ll}
A & B \\
C & D
\end{array}\right)=\left(\begin{array}{rr}
0 & 1 \\
-1 & 0
\end{array}\right)
$$

shows that the modular group is a subgroup of $\operatorname{Sp}(2 \mathrm{~g}, \mathbb{Z})$. Equation (34) is equivalent to

$$
A^{T} C=C^{T} A, \quad B^{T} D=D^{T} B, \quad A^{T} D-C^{T} B=1 .
$$

Under a modular transformation $\left(\begin{array}{ll}A & B \\ C & D\end{array}\right)$, the period matrix changes by

$$
\Omega \rightarrow \Omega^{\prime}=(A \Omega+B)(C \Omega+D)^{-1} .
$$

It follows that

$$
\left(\operatorname{Im} \Omega^{\prime}\right)^{-1}=(C \Omega+D)(\operatorname{Im} \Omega)^{-1}\left(\bar{\Omega} C^{T}+D^{T}\right) .
$$

Therefore, the finite temperature piece of the action $\Delta S(\beta ; \vec{m}, \vec{n})$ transforms under $\left(\begin{array}{ll}A & B \\ C & D\end{array}\right)$ by

$$
\begin{aligned}
\Delta S(\beta ; \vec{m}, \vec{n}) & =\frac{\beta^{2} T}{2}(\vec{m} \cdot \Omega-\vec{n})(\operatorname{Im} \Omega)^{-1}(\vec{m} \cdot \Omega-\vec{n})^{\dagger} \\
& \rightarrow \frac{\beta^{2} T}{2}\left(\vec{m} \cdot \Omega^{\prime}-\vec{n}\right)\left(\operatorname{Im} \Omega^{\prime}\right)^{-1}\left(\vec{m} \cdot \Omega^{\prime}-\vec{n}\right)^{\dagger} \\
& =\frac{\beta^{2} T}{2}\left(\vec{m}^{\prime} \cdot \Omega-\vec{n}^{\prime}\right)(\operatorname{Im} \Omega)^{-1}\left(\vec{m}^{\prime} \Omega-\vec{n}^{\prime}\right)^{\dagger} \\
& =\Delta S\left(\beta ; \vec{m}^{\prime}, \vec{n}^{\prime}\right),
\end{aligned}
$$

where

$$
\begin{aligned}
& \vec{m}^{\prime}=\vec{m} \cdot D-\vec{n} \cdot B, \\
& \vec{n}^{\prime}=\vec{n} \cdot A-\vec{m} \cdot C .
\end{aligned}
$$


Equations (39) and (40) can be inverted using (35) to give

$$
\begin{aligned}
& \vec{m}=\vec{m}^{\prime} \cdot A^{T}+\vec{n}^{\prime} \cdot B^{T}, \\
& \vec{n}=\vec{n}^{\prime} \cdot D^{T}+\vec{m}^{\prime} \cdot C^{T} .
\end{aligned}
$$

Thus, for each $\left(\begin{array}{l}\vec{m} \\ \vec{n}\end{array}\right)$ there is a unique $\left(\begin{array}{l}\vec{m}^{\prime} \\ \vec{n}^{\prime}\end{array}\right)$. The effect of a modular transformation on $F_{\mathrm{g}}(\beta)$ is just to reorder the infinite sums, thereby changing the dummy variables in (32) from $m_{i}, n_{i}$ to $m_{i}^{\prime}, n_{i}^{\prime}$. This proves the modular invariance of the free energy at any genus.

The modular invariance of (32) can now be used to show that all windings can be put in a single homology cycle. Let us see under what conditions an arbitrary $\vec{m}, \vec{n}$ can be transformed into $\vec{m}=(1,0, \ldots, 0)$ and $\vec{n}=(0, \ldots, 0)$. Suppose all entries $m_{i}, n_{i}$ have a greatest common divisor (G.C.D.) $r$,

$$
m_{i}=r \mu_{i}, \quad n_{i}=r v_{i}, \quad r \geqq 1, \quad \mu_{i}, v_{i} \in \mathbb{Z} .
$$

The first component of (41) becomes

$$
1=r\left(A_{i j} \mu_{j}+B_{i j} v_{j}\right)
$$

Both $r$ and the factor in parentheses are integers, so each must individually be equal to one. This shows that $\left(\begin{array}{l}\vec{m} \\ \vec{n}\end{array}\right)$ can be brought to the form $\left(\begin{array}{l}\vec{m}^{\prime} \\ \vec{n}^{\prime}\end{array}\right)=\left(\begin{array}{l}\delta_{1 i} \\ \overrightarrow{0}\end{array}\right)$ by a modular transformation only if all $m_{i}, n_{i}$ are relatively prime. As in the genus one case, the converse is also true. It then follows that there is always a modular transformation which takes an arbitrary $\left(\begin{array}{l}\vec{m} \\ \vec{n}\end{array}\right)$, not necessarily relatively prime, to $\left(\begin{array}{l}r \delta_{1 i} \\ \overrightarrow{0}\end{array}\right)$, where $r$ is the greatest common divisor of $m_{i}$ and $n_{i}$. A constructive proof will now be presented.

The proof that all non-trivial windings of the surface $M_{\mathrm{g}}$ embedded in $\mathbb{R}^{25} \times S^{1}$ can be put in one homology cycle proceeds by induction. The case $\mathrm{g}=1$ was proved in Sect. II. If the theorem holds at genus $g-1$, then it suffices to show that the windings can be moved from the gth to the $(g-1)$ th handle. In fact, the ordering of the handles is irrelevant ${ }^{2}$. The modular group transforming the homology cycles on those two handles is therefore a $\operatorname{Sp}(4, \mathbb{Z})$ subgroup of $\operatorname{Sp}(2 \mathrm{~g}, \mathbb{Z})$. This is equivalent to working on the genus 2 surface (i.e. ignoring all but two handles), so the relevant cycles will be labelled now $a_{1}, b_{1}, a_{2}, b_{2}$. The associated winding numbers are $m_{1}, n_{1}$, $m_{2}, n_{2}$.

First, all windings in handle 2 can be moved to the cycle $b_{2}$, say, using a transformation under the diagonal $\operatorname{Sp}(2, \mathbb{Z})$ subgroup of $\operatorname{Sp}(4, \mathbb{Z})$ which leaves $a_{1}, b_{1}$ fixed. If $m_{i}=r_{i} \mu_{i}, n_{i}=r_{i} v_{i}(i=1,2)$ with $\mu_{i}, v_{i}$ relatively prime, then the matrix $\left(\begin{array}{ll}1 & 0 \\ 0 & \gamma\end{array}\right)$, with 1 and $\gamma$ both $2 \times 2$ matrices, will do the job, where $\gamma=\left(\begin{array}{cc}v_{2} & -\mu_{2} \\ \tilde{\mu} & \tilde{v}\end{array}\right)$ is of

2 The homology basis in what follows shall be ordered by handle $\left(a_{1}, b_{1}, \ldots, a_{\mathrm{g}}, b_{\mathrm{g}}\right)$ 
the form used at genus one. For example, this choice of $\gamma$ leads to

$$
\begin{aligned}
& a_{1} \rightarrow a_{1}^{\prime}=a_{1}, \quad b_{1} \rightarrow b_{1}^{\prime}=b_{1}, \\
& a_{2} \rightarrow a_{2}^{\prime}=v_{2} a_{2}-\mu_{2} b_{2}, \quad b_{2} \rightarrow b_{2}^{\prime}=\tilde{\mu} a_{2}+\tilde{v} b_{2}, \quad \tilde{\mu} \mu_{1}+\tilde{v} v_{2}=1 .
\end{aligned}
$$

Under (45), $b_{2}$ will obtain winding number $r_{2}$, the G.C.D. of $m_{2}, n_{2}$, just as for the torus.

For convenience, assume also that all windings in handle 1 have been moved to the cycle $b_{1}$, with winding number $r_{1}$. Let $r$ be the G.C.D. of $r_{1}$ and $r_{2}$, so $r_{1}=r \rho_{1}$, $r_{2}=r \rho_{2}$. Then the matrix

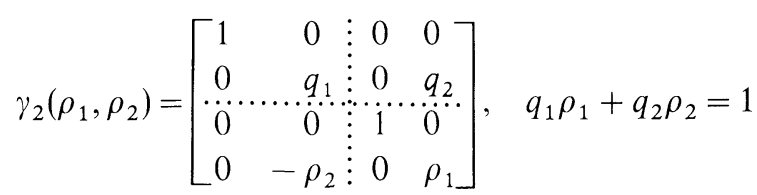

transforms all windings from $b_{2}$ to $b_{1}$, as promised, and satisfies (35). The topologically conserved winding number $r$ for the two handles is the greatest common divisor of the winding numbers $m_{1}, n_{1}, m_{2}, n_{2}$.

In fact, $(46)$ is not the most general form of a $\operatorname{Sp}(4, \mathbb{Z})$ matrix which unwinds the second handle. The matrix $\left(\begin{array}{ll}A & B \\ C & D\end{array}\right)$ can have arbitrary integer entries subject to the conditions (35) and $\operatorname{det} \gamma_{2}=1$. Four degrees of freedom remain, in addition to the freedom in how one chooses $q_{1}, q_{2}$. This allows

$$
\gamma_{2}\left(\rho_{1}, \rho_{2}\right)=\left[\begin{array}{lrr:cc}
1 & 0 & \vdots & B_{11} & 0 \\
A_{21} & q_{1} & B_{21} & q_{2} \\
\hdashline & 0 & \vdots & 1 & 0 \\
0 & -\rho_{2} & D_{21} & \rho_{1}
\end{array}\right] .
$$

This procedure can be repeated on $M_{\mathrm{g}}$ until all windings reside in one cycle. By a diagonal $[S p(2, \mathbb{Z})]^{g}$ transformation, windings for all the handles are put in the $b$ cycles. Then a sequence of $\operatorname{Sp}(4, \mathbb{Z})$ transformations moves them ultimately, say, to $a_{1}$, with winding number $r$ the G.C.D. of all the $m_{i}, n_{i}$. The product of these transformations will be a modular transformation $\gamma=\left(\begin{array}{ll}A & B \\ C & D\end{array}\right)$ such that $\gamma\left(\begin{array}{l}\vec{m} \\ \vec{n}\end{array}\right)=$ $\left(\begin{array}{l}r \delta_{1 i} \\ \overrightarrow{0}\end{array}\right)$. Clearly, this $\gamma$ is not unique since there exist many choices at each step of the procedure.

Let $\mathscr{G}_{1}$ be the subgroup of the modular group $\Gamma_{g}$ which leaves $\left(\begin{array}{l}r \delta_{1 i} \\ \overrightarrow{0}\end{array}\right)$ invariant. Then for any $\alpha \in \mathscr{G}_{1}, \alpha \circ \gamma\left(\begin{array}{l}\vec{m} \\ \vec{n}\end{array}\right)=\left(\begin{array}{l}r \delta_{1 i} \\ \overrightarrow{0}\end{array}\right)$, if $\gamma\left(\begin{array}{l}\vec{m} \\ \vec{n}\end{array}\right)=\left(\begin{array}{l}r \delta_{1 i} \\ \overrightarrow{0}\end{array}\right)$. Moreover, if $\gamma_{1}$ and $\gamma_{2}$ both take $\left(\begin{array}{l}\vec{m} \\ \vec{n}\end{array}\right)$ to $\left(\begin{array}{l}r \delta_{1 i} \\ \overrightarrow{0}\end{array}\right)$, then $\alpha=\gamma_{1} \gamma_{2}^{-1} \in \mathscr{G}_{1}$. We thus conclude that for every set of $2 \mathrm{~g}$ integers $m_{i}, n_{i}$, there is a unique $\gamma \in \Gamma_{\mathrm{g}} / \mathscr{G}_{1}$ which takes $\left(\begin{array}{l}\vec{m} \\ \vec{n}\end{array}\right)$ to $\left(\begin{array}{l}r \delta_{1 i} \\ \overrightarrow{0}\end{array}\right)$. 
Consider the action of the group $\mathscr{G}_{1}$ on the homology basis $\left(\begin{array}{c}\vec{a} \\ \vec{b}\end{array}\right)$. By comparing (33) with (41) and (42), one sees that

$$
\begin{aligned}
& a_{1}^{\prime}=a_{1}+\left(\text { linear combination of } a_{2}, \ldots, a_{\mathrm{g}}, b_{1}, \ldots, b_{\mathrm{g}}\right), \\
& a_{j}^{\prime}=\left(\text { Independent of } a_{1}\right) j \neq 1, \\
& b_{i}^{\prime}=\left(\text { Independent of } a_{1}\right) i=1, \ldots, \mathrm{g} .
\end{aligned}
$$

Furthermore, all modular transformations which satisfy (48) are elements of $\mathscr{G}_{1}$. So $\mathscr{G}_{1}$ can alternatively be defined as the subgroup of $\Gamma_{g}$ which preserves winding around the cycle $a_{1}$ only.

Our main result can now be proven. In the sums appearing in the expression for the free energy

$$
\left.F_{\mathrm{g}}(\beta)=\sum_{\vec{m}, \vec{n}}^{\prime} \int_{\mathscr{T} / \Gamma_{\mathrm{g}}}(d \tau) I_{\mathrm{g}}(\tau) \exp -\frac{\beta^{2} T}{2}[\vec{m} \cdot \Omega-\vec{n})(\operatorname{Im} \Omega)^{-1}(\vec{m} \cdot \Omega-\vec{n})^{\dagger}\right]
$$

make the change of variables

$$
\left(\begin{array}{l}
\vec{m} \\
\vec{n}
\end{array}\right)=r \gamma \circ\left(\begin{array}{l}
\delta_{1 i} \\
\overrightarrow{0}
\end{array}\right)
$$

where $r$ is the G.C.D. of $m_{i}, n_{i}$ and $\gamma \in \Gamma_{\mathrm{g}} / \mathscr{G}_{1}$.

As $\left(\begin{array}{l}\vec{m} \\ \vec{n}\end{array}\right)$ runs over all $2 \mathrm{~g}$-tuples of integers, $r$ runs over $\mathbb{Z}$ and $\gamma$ runs over all of $\Gamma_{\mathrm{g}} / \mathscr{G}_{1}$ once and only once. Then $F_{\mathrm{g}}(\beta)$ becomes

$$
F_{\mathrm{g}}(\beta)=\sum_{r=1}^{\infty} \sum_{\gamma \in \Gamma_{\mathrm{g}} / \mathscr{G}_{1}} \int_{\mathcal{T} / \Gamma_{\mathrm{g}}}(d \tau) I_{\mathrm{g}}(\tau) \exp -\frac{\beta^{2} T}{2} r^{2}[\operatorname{Im}(\gamma \circ \Omega)]_{1,1}^{-1}
$$

where the subscript 1,1 refers to that component of the matrix, and the action of $\gamma$ on $\Omega$ is given by (36). The change of variable

$$
(\text { moduli }) \rightarrow \gamma \circ(\text { moduli })
$$

in the integration over moduli space yields

$$
F_{\mathrm{g}}(\beta)=\sum_{r=1}^{\infty} \sum_{\gamma \in \Gamma_{\mathrm{g}} / \mathcal{S}_{1}} \int_{\gamma \circ\left(\mathscr{T} / \Gamma_{\mathrm{g}}\right)}(d \tau) I_{\mathrm{g}}(\tau) \exp -\frac{\beta^{2} T}{2} r^{2}[\operatorname{Im} \Omega]_{1,1}^{-1} .
$$

The modular invariance of $(d \tau)_{\mathrm{WP}}$ and $I_{\mathrm{g}}(\tau)$ have been used to obtain (53). Note that an element $\gamma \in \Gamma_{\mathrm{g}}$ has a natural action on $\mathscr{T} / \Gamma_{\mathrm{g}}: \gamma$ carries a point in one fundamental domain of $\mathscr{T} / \Gamma_{\mathrm{g}}$ to the corresponding point in a different fundamental domain.

The sum over $\gamma$ in (53) is performed by Poincaré resummation, as in the Selberg trace formula [6]:

$$
F_{\mathrm{g}}(\beta)=\sum_{\mathrm{r}=1}^{\infty} \int_{\mathscr{T} / \mathscr{G}_{1}}(d \tau) I_{\mathrm{g}}(\tau) \exp -\frac{\beta^{2} T}{2} r^{2}[\operatorname{Im} \Omega]_{1,1}^{-1}
$$

This is the desired result. Equation (54) can be obtained directly from the path 
integral by imposing the boundary conditions that $X^{0}(z, \bar{z})$ has winding number $r$ around the cycle $a_{1}$ and is strictly periodic around all the other cycles. $\mathscr{G}_{1}$ is the subgroup of $\Gamma_{\mathrm{g}}$ which preserves this boundary condition. Clearly the choice of $a_{1}$ is arbitrary; the derivation of (54) would remain valid with $a_{1}$ replaced by (say) $b_{3}$.

The expression (54) for the free energy at the g-loop level has the advantages over (32) in that:

(i) All but one of the $2 \mathrm{~g}$ infinite sums have been eliminated.

(ii) The region of integration $\mathscr{T} / \mathscr{G}_{1}$ may be simpler in practice than $\mathscr{M}=\mathscr{T} / \Gamma_{\mathrm{g}}$. Although the manifest modular invariance of $F_{\mathrm{g}}(\beta)$ has been sacrificed, the derivation of (54) from (32) makes it clear that these symmetries are still intact

\section{Conclusions}

The free energy for a gas of interacting closed bosonic strings has been calculated using the Polyakov path integral. Thermal effects introduce various winding numbers for the field $X^{0}(z, \bar{z})$ mapping the string worldsheet $M_{\mathrm{g}}$ around the compact time direction. It has been proven that the manifestly modular invariant formulation is equivalent to one putting all the winding into one arbitrary non-trivial closed curve. The latter formulation yields more readily to direct computation.

After this work was completed, we received a paper by Ohrndorf [12] which derives the generalization of our classical action for the embedding of higher genus surfaces in a compactified spacetime $\mathbb{R}^{26-D} \times \Lambda^{D}$, where $\Lambda^{D}$ is a lattice of dimension $D$. Upon judicious placement of lattice vector indices on our variables $\beta, m_{i}, n_{i}, H_{i}$ and choosing a metric on $\Lambda^{D}$ to contract indices, Ohrndorf's result can be obtained. Ohrndorf does not, however, discuss the consequences of modular invariance for this result.

Ultimately, our description of early string cosmology will hinge upon our ability to calculate non-perturbatively in the infinite genus limit [13] corresponding to a dense gas of strings. Of course, this is the physical regime in which the loop approximation in the sigma model breaks down, i.e. $\mathrm{g}_{\mu \nu}(x) \neq \delta_{\mu \nu}$. The assumptions which justify the free string calculations of the Hagedorn temperature no longer hold. The effects of higher genus surfaces and curvature are under investigation.

\section{Appendix I}

Beginning from the form of the free energy in (9), the critical temperature is calculated. Omitting an overall constant and the $T^{13}$, the $\tau$ integration in (9) can be written

$$
\mathscr{I}=\int_{-1 / 2}^{1 / 2} d \tau_{1} \int_{0}^{\infty} d \tau_{2} \tau_{2}^{-14} e^{4 \pi \tau_{2}}\left|f\left(e^{2 \pi i \tau}\right)\right|^{-48} \sum_{r=1}^{\infty} e^{-r^{2} \beta^{2} T / 2 \tau_{2}}
$$

The $\tau_{2}$ integral can be evaluated in the asymptotic limit corresponding to large mass level, $n \rightarrow \infty$. The function $f\left(e^{2 \pi i \tau}\right) \equiv f(x)$ in $(10)$ can be written with $x=e^{2 \pi i \tau}$ $=e^{2 \pi i \tau_{1}} e^{-2 \pi \tau_{2}}$ as

$$
|f(x)|^{-48} \equiv\left|\prod_{n=1}^{\infty}\left(1-x^{n}\right)\right|^{-48}=\left|1+\sum_{n=1}^{\infty} p(n) x^{n}\right|^{48} .
$$


The number $p(n)$ is the degeneracy of the $n$th mass level. For $-\frac{1}{2}<\tau_{1}<\frac{1}{2}$, the absolute value is dominated by the value at $\tau_{1}=0$. Hence

$$
\begin{aligned}
\left|1+\sum_{n=1}^{\infty} p(n) x^{n}\right|^{48} & \leqq\left[1+\sum_{n=1}^{\infty} p(n) e^{-2 \pi \tau_{2} n}\right]^{48} \\
& \equiv 1+\sum_{n=1}^{\infty} p_{48}(n) e^{-2 \pi \tau_{2} n}
\end{aligned}
$$

where $p_{48}(n)$ is given asymptotically by Huang and Weinberg [14]:

$$
p_{48}(n) \sim \frac{1}{\sqrt{2}} 2^{49 / 4} n^{-51 / 4} \exp 2 \pi\left(\frac{48 n}{6}\right)^{1 / 2} \sim 2^{47 / 4} n^{-51 / 4} \exp 4 \pi(2 n)^{1 / 2},
$$

for $n \gtrsim n_{0}$, for some $n_{0}$. Considering those terms for which $n>n_{0}$, one has asymptotically

$$
\begin{aligned}
\mathscr{I} & \sim \text { const } \int_{0}^{\infty} d \tau_{2} \tau_{2}^{-14} \sum_{n=n_{0}}^{\infty} p_{48}(n) e^{-2 \pi \tau_{2}(n-2)} \sum_{r=1}^{\infty} e^{-r^{2} \beta^{2} T / 2 \tau_{2}} \\
& =\text { const } \sum_{n=n_{0}}^{\infty} \sum_{r=1}^{\infty} p_{48}(n)\left(\frac{r^{2} \beta^{2} T}{4 \pi(n-2)}\right)^{-13 / 2} K_{13}(r \beta \sqrt{4 \pi(n-2) T}),
\end{aligned}
$$

where $K_{13}$ is a modified Bessel function. For large $n, K_{13}$ behaves as

$$
K_{13}\left(r \beta \sqrt{4 \pi(n-2) T)} \underset{n \rightarrow \infty}{\sim} \sqrt{\frac{\pi}{2}}(r \beta \sqrt{4 \pi(n-2) T})^{-1 / 2} \exp -r \beta \sqrt{4 \pi(n-2) T} .\right.
$$

Since for large $n,(n-2) \sim n$, using (A.4) gives

$$
\mathscr{I} \sim \text { const } \sum_{n=n_{0}}^{\infty} \sum_{r=1}^{\infty} n^{-13 / 2}\left(r^{2} \beta^{2} T\right)^{-27 / 4} e^{4 \pi \sqrt{2 n}} e^{-r \beta \sqrt{4 \pi n T}} .
$$

The dominant contribution comes from the $r=1 \mathrm{term}$, so the critical temperature obtains by equating the exponents:

$$
\beta_{c}=\sqrt{\frac{8 \pi}{T}}
$$

This agrees with the known result. Note that only an upper bound on $\beta_{c}$ has been calculated, i.e., a lower bound on the critical temperature, because of the argument leading to (A.3).

\section{Appendix II}

The divergences of $F_{1}(\beta)$ below the critical temperature are examined here at each mass level $n$, using $\left|1+\sum p(n) x^{n}\right|^{48}=1+\sum p_{48}(n) x^{n}$. The lowest mass level is the tachyon, corresponding to $n=0$, where the 1 in (A.2) can be written $1=p(0) x^{0}$. This contributes a factor

which diverges like $e^{4 \pi \tau_{2}}$ as $\tau_{2} \rightarrow \infty$.

$$
\sum_{r=1}^{\infty} \int_{0}^{\infty} d \tau_{2} \tau_{2}^{-14} e^{4 \pi \tau_{2}} e^{-r^{2} \beta^{2} T / 2 \tau_{2}}
$$


At $n=1$, one would guess that the dilaton behavior is like

$$
\sum_{r=1}^{\infty} \int_{0}^{\infty} d \tau_{2} \tau_{2}^{-14} e^{4 \pi \tau_{2}} p_{48}(1) e^{-2 \pi \tau_{2}} e^{-r^{2} \beta^{2} T / 2 \tau_{2}}
$$

However, $|f(x)|^{-48}$ can be expanded as

$$
\begin{aligned}
\left|1+\sum_{n=1}^{\infty} p(n) x^{n}\right|^{48} & =\left(1+\sum_{n^{\prime}=1}^{\infty} p\left(n^{\prime}\right) x^{n^{\prime}}\right)^{24}\left(1+\sum_{n^{\prime \prime}=1}^{\infty} p\left(n^{\prime \prime}\right) x^{* n^{\prime \prime}}\right)^{24} \\
& =1+24 p(1)\left(x+x^{*}\right)+O\left(|x|^{2}\right)+\cdots \\
& =1+24 e^{-2 \pi \tau_{2}}\left(e^{2 \pi i \tau_{1}}+e^{-2 \pi i \tau_{1}}\right)+\cdots
\end{aligned}
$$

Keeping in mind the integration $\int_{-1 / 2}^{1 / 2} d \tau_{1}$ in (A.1), one sees that the terms of first order in $x$ vanish because of periodicity in $\tau_{1}$. Consequently, there is no dilation divergence.

For $n>1$, the expansion (A.9) will include interference terms from various $n^{\prime}<n$. Again, the phase integrals vanish expect for the terms in which the phases sum to zero, i.e. $\left(x^{n / 2}\right)\left(x^{* n / 2}\right)$, for even $n$. For the level $n=2$ this interference term contributes a factor

$$
\begin{aligned}
& \int_{-1 / 2}^{1 / 2} d \tau_{1} \int_{0}^{\infty} d \tau_{2} \tau_{2}^{-14} e^{4 \pi \tau_{2}} e^{-4 \pi \tau_{2}} e^{-r^{2} \beta^{2} T / 2 \tau_{2}}\left(576 e^{2 \pi i \tau_{1}} e^{-2 \pi i \tau_{1}}\right) \\
& =576\left(\frac{r^{2} \beta^{2} T}{2}\right)^{-13} \Gamma(13) .
\end{aligned}
$$

This makes a finite contribution to $F_{1}(\beta)$. All higher order terms will also be finite. However, only for $\beta>\beta_{c}$ does the sum over $r$ converge.

Acknowledgements. We are grateful for helpful discussions with J. Polchinski, S. Blau, M. Clements, S. Carlip and S. Della Pietra. Special thanks also to D. Eades and A. Traverso. B. D. B. R. is most appreciative for the encouragement and support from S. Weinberg. Supported in part by the Robert A. Welch Foundation and NSF Grant 8605978. B. McClain is also supported in part by NSF 8405648.

Note Added. After this paper was accepted for publication, we became aware of similar results obtained by K. H. O'Brien and C.-I. Tan, Brown University preprint, Brown-HET-602.

\section{References}

1. Polchinski, J.: Commun. Math. Phys. 104, 37 (1986)

2. Alvarez, E.: Strings at finite temperature. CERN preprint CERN-TH.4262/85; Bowick, M. J., Wijewardhana, L. C. R.: Phys. Rev. Lett. 54, 2485 (1985), Gen. Rel. Grav. 18, 59 (1986); Okada, H.: Tokyo preprint TIT/HEP-92, 1986; Oleson, P.: On the exponentially increasing level density in string models and the Tachyon singularity. CERN preprint CERN-TH.4249/85; Sundborg, B.: Nucl. Phys. B254, 583 (1985); Tye, S. H. H.: Phys. Lett. 158B, 388 (1985)

3. Hagedorn, R.: Nuovo Cimento [Suppl.] 3, 147 (1965) and Cargese Lectures in Physics, Vol. 6. Schatzman, E. (ed.) New York: Gordon and Breach 1973, p. 643

4. Moore, G.: Modular forms and two-loop string physics. Harvard preprint HUTP-86/A038

5. Polyakov, A. M.: Phys. Lett. 103B, 207 (1981); Alvarez, O.: Nucl. Phys. B216, 125 (1983); Moore, G., Nelson, P.: Nucl. Phys. B266, 55 (1986) 
6. D'Hoker, E., Phong, D.: Nucl. Phys. B269, 205 (1986)

7. Bernard, C. W.: Phys. Rev. D 9, 3312 (1974)

8. Alvarez-Gaumé, L.: Topological methods in string perturbation theory. CERN preprint CERNTH. $4480 / 86$

9. Alvarez-Gaumé, L., Moore, G., Vafa, C.: Commun. Math. Phys. 106, 1 (1986)

10. Fay, J. D.: Lecture Notes in Mathematics, Vol. 352 Berlin, Heidelberg, New York: Springer 1973

11. Nelson, P.: Lectures on strings and moduli space. Harvard preprint HUTP-86/A047

12. Ohrndorf, T.: On the compactification of the Bosonic string at higher loops. CERN preprint CERNTH. $4425 / 86$

13. Friedan, D.: A new formulation of string theory. Chicago preprint EFI 86-52

14. Huang, K., Weinberg, S.: Phys. Rev. Lett. 25, 895 (1970)

Communicated by S.-T. Yau

Received December 29, 1986; in revised form February 19, 1987 